\title{
LARGE-SCALE AVALANCHE BRAKING MOUND AND CATCHING DAM EXPERIMENTS WITH SNOW: A STUDY OF THE AIRBORNE JET
}

\author{
KRISTIN MARTHA HÁKONARDÓTTIR \\ The Icelandic Meteorological Office, Bustadavegi 9, 150 Reykjavik, Iceland \\ E-mail: k.hakonardottir@bristol.ac.uk \\ ANDREW J. HOGG \\ Centre for Environmental \& Geophysical Flows, School of Mathematics, University of Bristol, \\ University Walk, Bristol BS8 1TW, U.K. \\ TÓMAS JÓHANNESSON \\ The Icelandic Meteorological Office, Bústadavegur 9, IS-150 Reykjavik, Iceland \\ MARTIN KERN and FELIX TIEFENBACHER \\ Swiss Federal Institute of Snow and Avalanche Research, Flüelstrasse 11, CH-7260 Davos Dorf, \\ Switzerland
}

(Received 5 December 2002; Accepted 15 March 2003)

\begin{abstract}
We report a series of ongoing large-scale experiments to study the interaction of a snow avalanche with a dam and a row of mounds which are of a comparable height to the flow depth. The experimental results indicate that the behaviour of the supercritical flow around the obstacles is governed by the large-scale properties of the flowing avalanche rather than micro-scale properties of the granular current. The experiments show that, similarly to smaller-scale experiments with glass particles, the avalanche detaches from the top of the dam or mound and forms a coherent airborne jet, which can be modelled as a two dimensional ballistic projectile with negligible air resistance. We study the two parameters that define the trajectory of the jet, namely the speed at which the jet is launched from the top of the obstacle and the deflection of the jet by the obstacle, and compare the results with a theory for the deflection of a jet of an ideal fluid.
\end{abstract}

Keywords: avalanches, braking mounds, catching dam, high Froude number flow, jet, large-scale snow experiments, launch angle, launch speed

\section{Introduction}

A series of large-scale experiments were performed to study the retarding effect of avalanche catching dams, and braking mounds, on an incoming snow avalanche. (Here, and throughout this paper, we use the terms dams and braking mounds to denote obstacles that do and do not span the width of the avalanche, respectively.) The experiments were performed in a large chute, situated on the mountainside Weissfluhjoch above the town of Davos in Switzerland. The experimental chute was built in the late 1950's to study impact forces on obstacles. Arrays of optical velocity sensors have recently been installed in the base of the chute as well as on 
the sides to obtain basal velocities and vertical velocity profiles through the depth of the avalanche (Tiefenbacher and Kern, in press).

The aim of this set of experiments was to analyse the flow over dams and over and around braking mounds, and compare the results with experimental results obtained at smaller scales, where small glass particles were used as the experimental material (Hákonardóttir et al., 2001, 2003). Experiments on several different scales have been carried out in order to establish design guidelines for avalanche braking mounds. The experiments were conducted as a part of the European Union project, Cadzie, during 1999-2002 in a collaboration between the Icelandic Meteorological Office, the School of Mathematics at the University of Bristol in England and the Swiss Federal Institute for Snow and Avalanche Research (SLF).

The snow-avalanche experiments showed that the flow was launched from the top of the dams and mounds and travelled a considerable distance before landing back on the slope (Figure 1). Images from video recordings of the experiments showed that the airborne jets could be modelled as a projectile motion in two dimensions with negligible air resistance. The formation of such an airborne jet has important practical consequences for the use of multiple rows of defence measures to retard avalanches. The spacing between the rows must be chosen sufficiently large so that the material launched from the first row does not jump over subsequent rows further down the slope. We report here the first results of these experimental studies. The snow-avalanche experiments at Weissfluhjoch are a matter of ongoing research and the need for more data is profound.

In this study we analyse the large-scale snow experiments by documenting the airborne jets which arise from the interaction of the avalanches with catching dams and braking mounds and by studying the two parameters that define the trajectory of the jets. The observed launch angle is compared with a theory for the deflection of a jet of an ideal fluid (Section 3.1), and the launch speed is studied by looking at the energy dissipation involved in the impact with the dams (Section 3.2).

\section{Experimental Setup and Design}

The experimental chute was $34 \mathrm{~m}$ long and $2.5 \mathrm{~m}$ wide with $0.9 \mathrm{~m}$ high side walls, see Figure 2. A volume of approximately $5 \mathrm{~m}^{3}$ of snow was released instantaneously from the top of the chute, by the rapid removal of a supporting lock gate. The snow had been shovelled manually into a barrel and then dumped onto the chute, behind the lock gate. After release, it flowed down the chute and carried on down the mountainside until finally coming to a rest on the skislope below. Rubber bars were placed on the base of the chute between the lock gate and the barriers to agitate the flow. A row of obstacles was placed in the flow path of the avalanche, perpendicular to the experimental chute and the progression of the avalanche down the chute was recorded using two video cameras. The flow speed at different locations upstream and downstream of the obstacles was also recorded using optical 


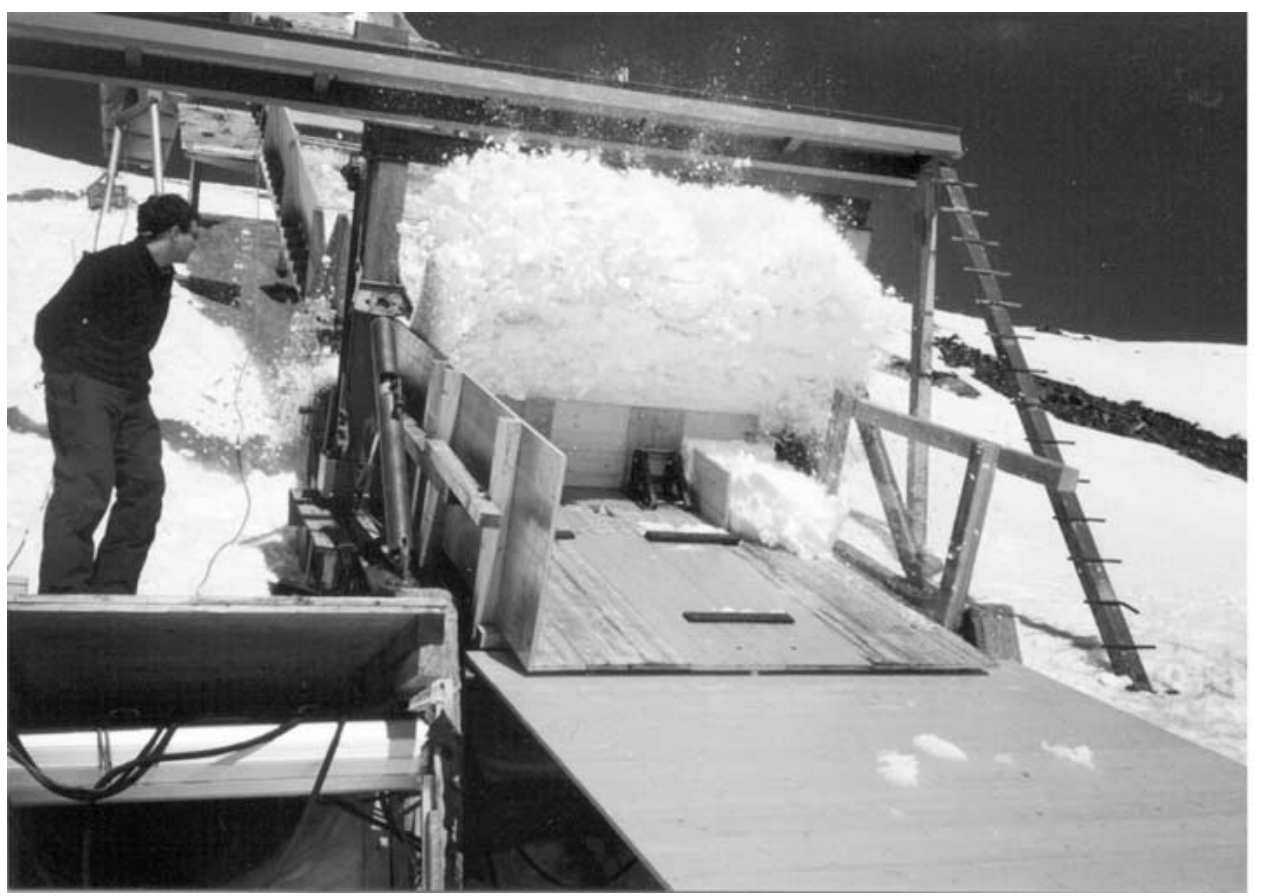

Figure 1. A photograph of an avalanche launched from a $60 \mathrm{~cm}$ high dam (and the right hand corner of the dam breaking). Photo: Andrew Hogg.

velocity sensors, see McElwaine and Tiefenbacher (2003) and Tiefenbacher and Kern (in press) for a description of the sensors.

The obstacles were either 0.4 or $0.6 \mathrm{~m}$ high, and the depth of the flow was estimated to be $0.3 \pm 0.1 \mathrm{~m}$. The catching dams spanned the entire width of the chute, while a row of mounds comprised two mounds, which only partially spanned the chute. The mounds had aspect ratios, defined in this context as the ratio of the height of the mound to its breadth, of 0.7 and 1.0 for the 0.4 and $0.6 \mathrm{~m}$ high mounds, respectively.

Large, dry, natural snow avalanches often consist of a powder cloud and a denser core. A typical Froude number of the dense core of such avalanches is of order 10 (McClung and Schaerer, 1993). In this context we define the Froude number by

$$
\operatorname{Fr}=\frac{u}{\sqrt{g \cos (\xi) h}}
$$

where $u$ is the speed of the flow, $g$ is the gravitational acceleration, $\xi$ is the inclination of the chute and $h$ is the flow depth. This dimensionless number is used to characterise free surface fluid flows. The design of the experiments and previous experiments at smaller scales was based on the conjecture that a Froude number of the same order of magnitude would approximately maintain dynamical similarity between natural snow avalanches and the smaller scale experimental avalanches. 


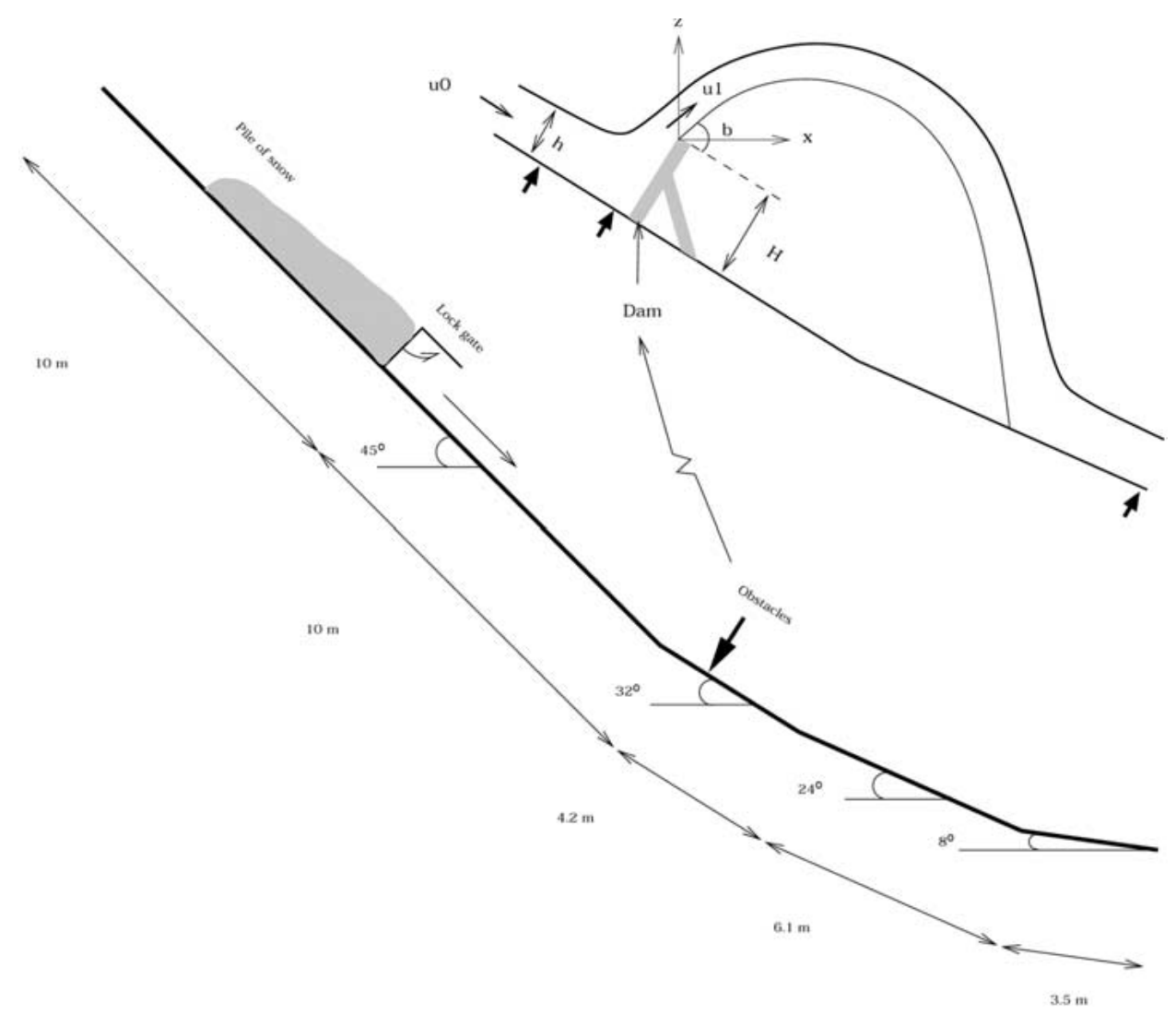

Figure 2. A schematic diagram of the experimental chute and a jet jumping over a dam on the chute, side view. The speeds $u_{0}$ and $u_{1}$ are defined, along with the launch angle, $\beta$, the depth of the flow, $h$ and the height of the dam, $H$. The arrows denote a row or an array of optical velocity sensors, see Tiefenbacher and Kern (in press).

The Froude numbers in the snow experiments presented here were in the range $3-6$, varying with each experimental run, depending on the condition of the snow. The wetter the snow, the slower and thicker the avalanche. These were the highest Froude numbers that could be realised from the experimental setup, and they were somewhat lower than those of natural events.

A corresponding obstacle Froude number, $\mathrm{Fr}_{b}$, can be defined as

$$
\operatorname{Fr}_{b}=\frac{u}{\sqrt{g \cos (\xi) H}}
$$

where $H$ denotes the height of the obstacles. The snow experiments yielded obstacle Froude numbers in the range 2-4; these were in the same range as in the previous experiments on smaller scales (Hákonardóttir et al., 2001, 2003). 


\section{Experimental results}

The snow used in the experiments was coarse grained and varied from being dry to being rather wet; it contained fine snow along with snow lumps with a diameter up to $0.1 \mathrm{~m}$. Before the snow was released it extended 5 to $6 \mathrm{~m}$ behind the lock gate. After the release, the avalanche spread longitudinally on the chute and had a length of about $8 \mathrm{~m}$ before reaching the obstacles. A head that was about $0.1 \mathrm{~m}$ higher than the snow immediately following and a thin tail were detected from the video recordings. The head travelled with the maximum flow speed, $7.5 \pm 1.0 \mathrm{~m} \mathrm{~s}^{-1}$, which was then maintained for just under $1 \mathrm{~s}$ and so was the depth of the flow just behind the head, $0.3 \pm 0.1 \mathrm{~m}$.

Experiments with catching dams in the flow path of the avalanche resulted in jets launched from the top of the obstacles (Figure 2). The duration of each avalanche was short, so the geometry of the jets was dominated by the first front taking off from the top of the dams and a steady jet, as had been observed in the smaller scale experiments, did not have sufficient time to develop fully.

The trajectories taken by the jets were studied from video recordings for flows over the two dams by tracking the first front of the flow. By treating the jet as a two-dimensional ballistic projectile, without air resistance, an expression for the jet trajectory taking off a dam, positioned on a slope of $32^{\circ}$, may be written as a function of the launch angle measured relative to the slope, $\beta$, and the launch speed, $u_{1}$, and is given by

$$
z=x \tan \left(\beta-32^{\circ}\right)-\frac{1}{2} \frac{g x^{2}}{u_{1}^{2}} \frac{1}{\cos ^{2}\left(\beta-32^{\circ}\right)} .
$$

Launch angles and launch speeds were calculated indirectly by fitting parabolas to the observed jet trajectories using least square fits (Figure 3).

The jet over the $0.4 \mathrm{~m}$ high dam was not as coherent and well defined as for the $0.6 \mathrm{~m}$ high dam. That might be caused by the difference in the wetness of the snow, the snow being considerably wetter in the experiment with the higher dam. It was thus possible to draw a number of plausible trajectories through the jet launched from the dam (in contrast to only one through the jet over the $0.6 \mathrm{~m}$ high dam), resulting in different values of launch speeds and angles. In order to obtain a trajectory for the $0.4 \mathrm{~m}$ high dam, it was necessary to constrain the choice of a trajectory to one where the horizontal component of the launch speed corresponded to the horizontal front velocity of the jet as measured from the video recording. The observed trajectory for the $0.6 \mathrm{~m}$ high dam was much more clearly defined, and a parabola fitted through the middle of the jet led to a good agreement between the horizontal component of the launch speed (evaluated from the parabola fitted to the trajectory) and the horizontal speed measured directly from the video recording, see Table I.

There is no suggestion that air resistance is affecting the jets, i.e., the shape of the jet is well approximated by a parabola and the velocity in the horizontal- 


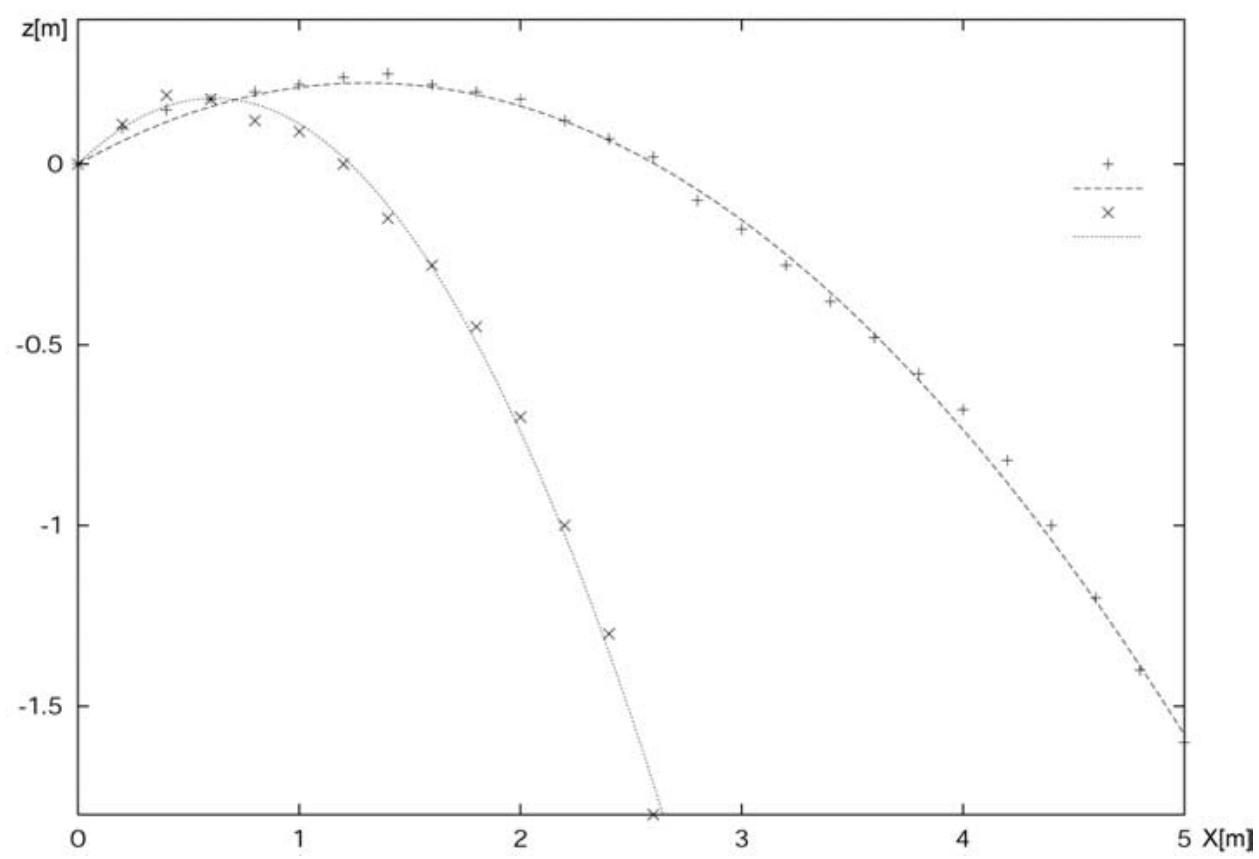

Figure 3. Jet trajectories for the two dams. The points show the observed trajectories and the lines denote the corresponding fitted parabolas used to calculate the launch speed and angle. The jet was not as coherent and well defined for the $0.4 \mathrm{~m}$ high dam as for the $0.6 \mathrm{~m}$ high dam. The length and height are measured from the tops of the dams, horizontally and vertically.

\section{TABLE I}

The incoming speed is noted by $u_{0}$ and was measured at an array of vertical velocity sensors placed at the side of the chute just in front of the dams, see Tiefenbacher and Kern (in press). The table shows the resulting launch speeds, $u_{1}$, and launch angles, $\beta$, for jets over the 0.4 and $0.6 \mathrm{~m}$ high dams. The accuracy range was obtained by comparing the horizontal part of the launch speed, $u_{1}$, with the horizontal speed of the first front of the jet, measured from the video recording.

\begin{tabular}{llll}
\hline$H(\mathrm{~m})$ & $u_{0}\left(\mathrm{~m} \mathrm{~s}^{-1}\right)$ & $\beta\left(^{\circ}\right)$ & $u_{1}\left(\mathrm{~m} \mathrm{~s}^{-1}\right)$ \\
\hline 0.4 & $7.5 \pm 1.5$ & 51 & $6.1 \pm 0.4$ \\
0.6 & $7.5 \pm 0.25$ & 63 & $3.7 \pm 0.1$ \\
\hline
\end{tabular}


direction, found from the analysis of the video recording, is approximately constant.

\subsection{JET DEFLECTION}

The inviscid irrotational flow over a dam has been studied theoretically when the effect of gravity is negligible (Yih, 1979). Using free-streamline theory and conformal mappings of the complex potential, it is possible to derive an expression for the launch angle of the jet as a function of the height of the dam relative to the depth of the flow and the inclination of the upstream dam face, $\alpha$ (here, $\alpha=\pi / 2$ ). For the jet defined by Figure 2, Yih (1979) derived the following expression for the height of the dam relative to the depth of the incoming stream

$$
\begin{aligned}
\frac{H}{h}= & \operatorname{Im}\left(\frac { 1 } { \pi } \sum _ { r = 0 } ^ { m - 1 } \left\{\exp (-i(2 r \alpha+\beta)) \ln \left[1-\exp \frac{i(\alpha-2 r \alpha-\beta)}{n}\right]\right.\right. \\
& -\exp (-i(2 r \alpha-\beta)) \ln \left[1-\exp \frac{i(\alpha-2 r \alpha+\beta)}{n}\right] \\
& \left.\left.+2 \exp (-i 2 r \alpha) \ln \left[1-\exp \frac{i(\alpha-2 r \alpha)}{n}\right]\right\}\right)
\end{aligned}
$$

where $\alpha=\pi n / m$. This expression provides an implicit relationship between the height of the dam relative to the flow depth and the launch angle. The theory predicts that the deflection of the jet asymptotically approaches the angle between the upstream face of the dam and the experimental chute (here $90^{\circ}$ ) as the height of the dam relative to the flow depth increases.

Figure 4 shows the launch angle plotted as a function of the nondimensional dam height for the two dams, along with the results from previous experiments on smaller scales for which glass particles were used and results from an experiment with fluid jets. Also shown is the theoretical prediction for a fluid jet. The snow avalanches seem to be following the same trend as the theory predicts, i.e. the current is deflected at a steeper angle as the dams become higher. The experimental results in general follow the theoretical curve for small values of the nondimensional dam height but, as $H / h$ increases, the launch angle for both fluid and granular flows approaches an angle that is $10^{\circ}-20^{\circ}$ lower than the angle between the experimental chute and the upstream dam face. A possible explanation for this difference is the formation of a deposited wedge of particles upstream of the dams.

After each experimental run we found snow deposited on the upstream faces of the dams, thus lowering the effective inclination of the face. This wedge was also seen on the smaller laboratory scales; it might be formed partially in the initial interaction with the dam, so that the jet is not deflected at the full angle of the upstream face of the dam for high dams. The wedge might also be partly built 


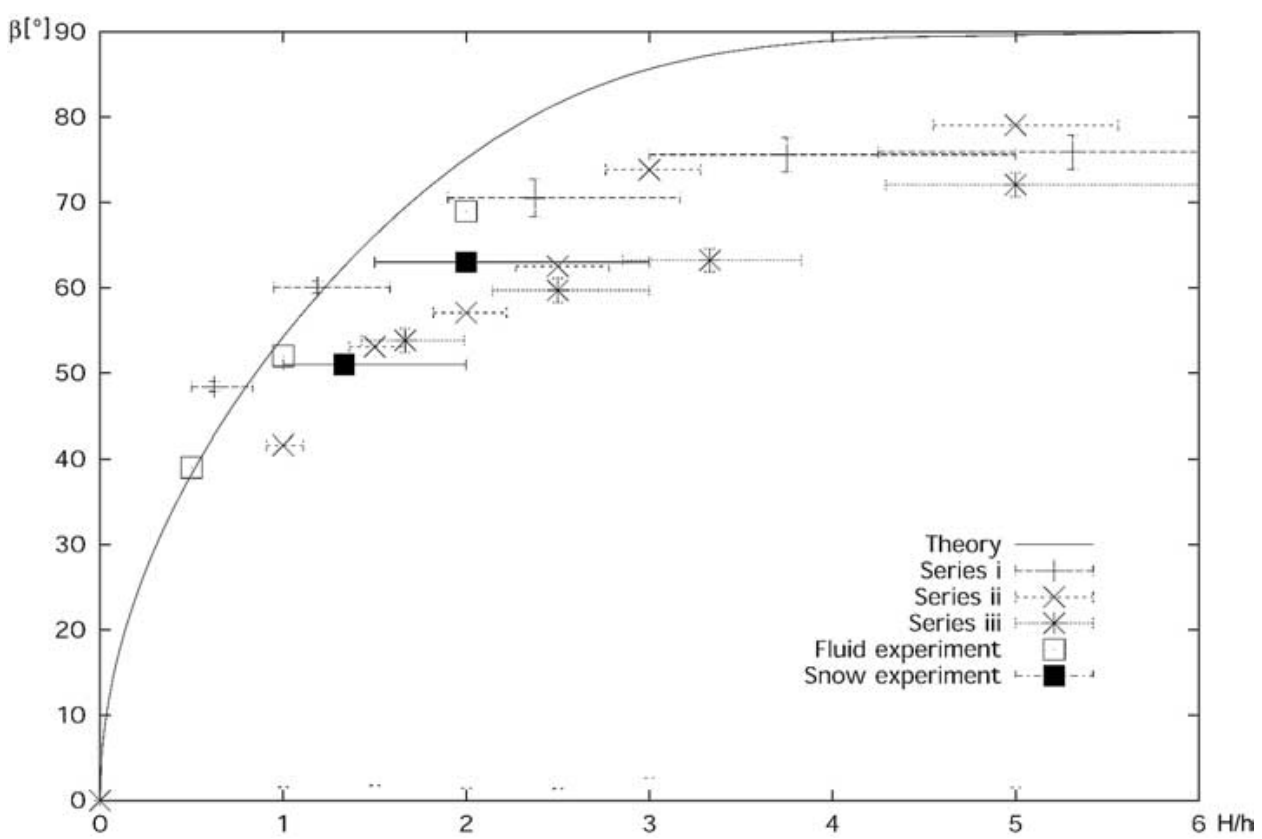

Figure 4. The launch angle, $\beta$, of a jet plotted as a function of the nondimensional dam height, $H / h$, for dams with an upstream face inclined at $\alpha=90^{\circ}$ to the experimental chute. Series (i), (ii), (iii) are experimental results on smaller scales where glass particles were used to form a supercritical granular flow. The solid line denotes the theory. Results from experiments using fluids from Yih (1979) are also shown.

up by the tail of the flow, flowing at lower speeds than the bulk of the avalanche. An experimental study of these wedges behind catching dams of varying heights and the so-called 'dead zones' behind the dams for lower Froude number flows $(2<\mathrm{Fr}<6)$ has been carried out by Faug et al. (2002). Numerical calculations of the dead zone behind an obstacle of an infinite height are described by Gray et al. (2003).

Similar wedges were observed behind the braking mounds, see Figure 5. The smaller scale experiments indicate that the dynamics of the interaction of granular flows with mounds is very similar to dams when the aspect ratio of the mound is small, i.e. most of the snow is launched straight over the mound. However, when the aspect ratio approaches unity, different mechanisms start to dominate the motion; most of the snow is deflected sideways over and around the mounds and coherent jet trajectories can no longer be observed. The video recordings of the snow experiments showed considerable lateral deflection of the jets of snow during the impact with the mounds. Wedges of this sort have also been observed behind boulders after pyroclastic flows from volcanic eruptions (Sparks et al., 2002). 


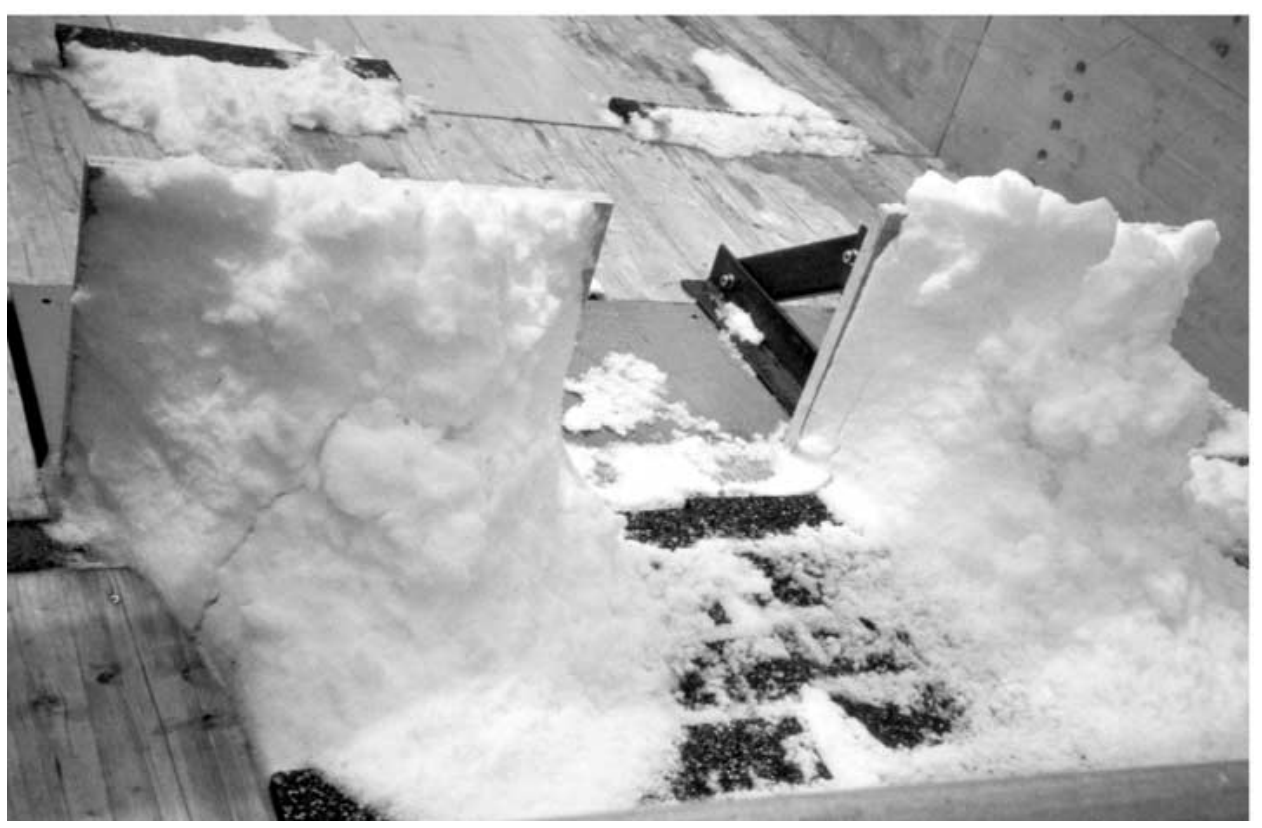

Figure 5. A photograph of wedges left behind the $0.4 \mathrm{~m}$ high mounds after an avalanche. Photo: Kristin Martha Hàkonardòttir.

\subsection{SPEED REDUCTION}

The energy dissipation involved in the impact of the avalanche and the obstacles can be analysed by plotting the ratio of the incoming speed and the launch velocity. If the mechanical energy of the flow is conserved in the collision, simple energy conservation gives

$$
\frac{1}{2} u_{1}^{2}=\frac{1}{2} u_{0}^{2}-g H^{\prime}
$$

where $H^{\prime}$ is the vertical rise of the flow when it passes over the obstacles $\left(H^{\prime}=\right.$ $H \cos 32^{\circ}$, since the dams were positioned on a slope of $32^{\circ}$ ). Hence, no energy loss during the impact with the obstacles leads to the expression

$$
\frac{u_{1}}{\sqrt{u_{0}^{2}-2 g H^{\prime}}}=1 .
$$

The ratio $D=u_{1} / \sqrt{u_{0}^{2}-2 g H^{\prime}}$ provides an estimate of the amount of energy dissipated in the turning process, since if the kinetic energy is solely converted into gravitational potential energy this ratio should be unity. We plot this ratio as a function of the obstacle height to flow depth in Figure 6. The figure suggests that there is considerable energy dissipation involved in the impact of the avalanche 


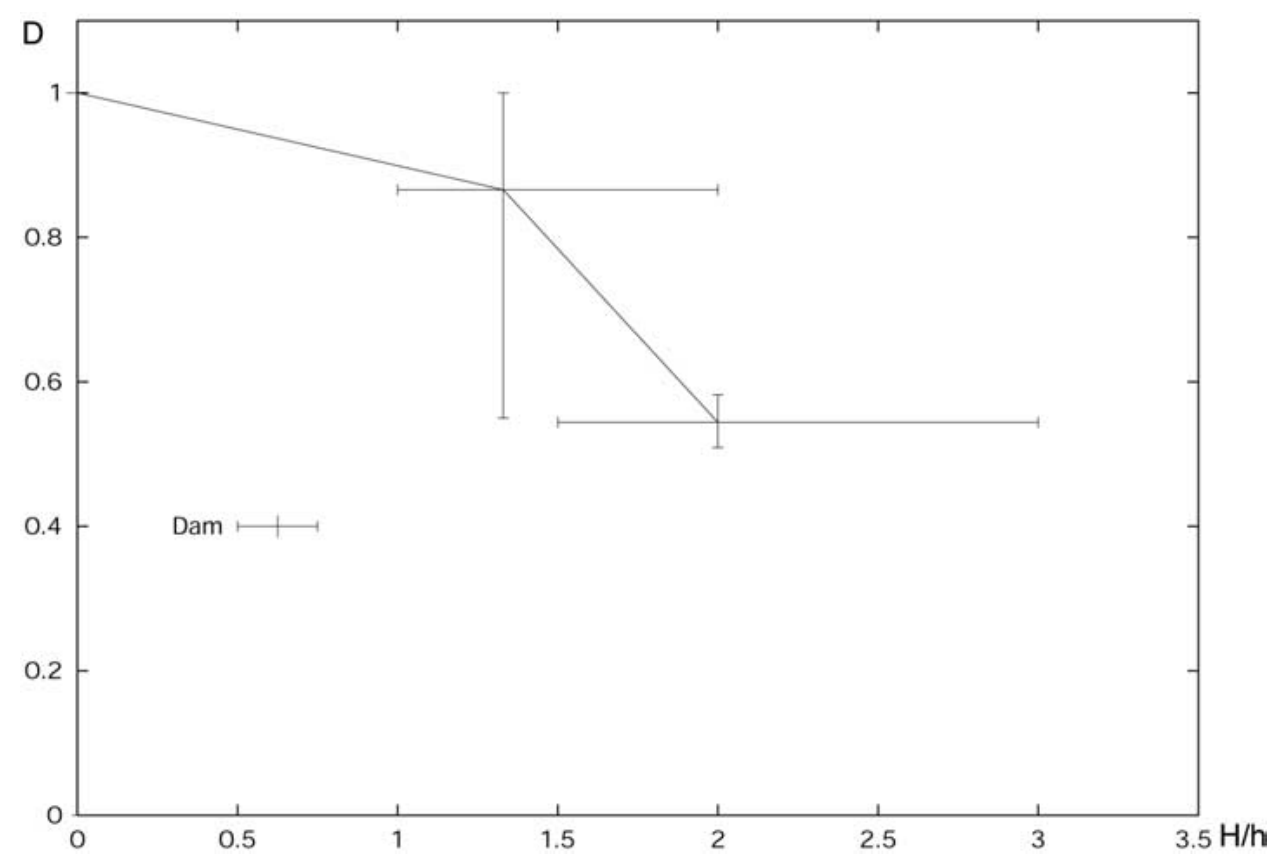

Figure 6. The relative velocity ratio, $D=u_{1} / \sqrt{u_{0}^{2}-2 g H \cos 32^{\circ}}$, plotted as a function of the nondimensional dam height, $H / h$, for the two dams.

with the dams. The high uncertainty in the speed associated with the lower dam makes it hard to distinguish between the effectiveness of the two dams.

It should be noted that the incoming speed, $u_{0}$, is measured at the side of the chute and could therefore be slightly lower than the speed at the centre of the avalanche, because of drag at the sides of the chute. The launch speed, $u_{1}$, is calculated from the observed trajectories and represents the speed of the centre of the avalanche. Also we do not account for any infill behind the dams, which could somewhat reduce the effective height of the dams, $H^{\prime}$, with the result that we may be under estimating the energy loss.

\section{Conclusions}

The main conclusion of this set of experiments is that similar features are observed in the interaction of the avalanches and the obstacles as were previously observed in experiments on much smaller scales using other granular materials. Jets of snow were launched from the top of the obstacles and subsequently landed back on the chute further down. The Froude number of the experiments was somewhat lower than believed to be appropriate for large natural snow avalanches; here the flows attained a Froude number in the range 3-6, rather than 10. Furthermore the thickness 
of the avalanche and the flow speed varied between the experiments depending on the wetness of the snow. In spite of the Froude number being lower than desired for dynamical similarity with large-scale avalanches and the variable conditions of the snow, the flow behaviour around the obstacles was similar to what was found in the smaller scale experiments with Froude numbers approximately equal to 10 . This suggests that the behaviour of the supercritical flow around the obstacles does not depend sensitively on the exact value of the Froude number. The similarity of the experimental results for different materials, and at different scales, furthermore indicates that the flow around the obstacles is primarily governed by the largescale properties of the flowing avalanche (i.e. flow depth and speed) rather than micro-scale properties of the granular current.

These experiments and previous experiments on smaller scales suggest the following behaviour of an avalanche hitting a catching dam and a row of braking mounds:

Dam. The snow hits the dam; subsequently it is launched from the top of the dam and forms a jet that lands further down on the chute. The jet can be treated as a two dimensional ballistic projectile. As the dam becomes higher, more energy is lost in the impact and the trajectory taken by the jet becomes steeper.

Braking mounds. The snow is deflected both over and around the mounds and an interaction between jets launched from adjacent mounds takes place. The wider the mounds become, compared to their height, the less significant/effective the lateral deflection becomes and the mounds behave more like dams. Energy dissipation takes place in the impact of the avalanche with the mounds and also in the interaction of jets launched from individual mounds.

The airborne jet that is formed by the collision of the flow with the obstacles has important practical consequences for the use of multiple rows of mounds or combinations of rows of mounds and a catching dam. The spacing between the rows must be chosen to be sufficiently large so that the material launched from the top of the obstacles does not jump over structures farther down the slope, in order for lower mounds and dams to have their full retarding effect.

The snow experiments on Weissfluhjoch are ongoing; additional data are needed to establish further the results reported here.

\section{References}

Faug, T., Lachamp, P., and Naaim, M.: 2002, 'Experimental Investigation on Steady Granular Flows Interfacing with an Obstacle Down an Inclined Channel: Study of the Dead Zone Upstream from the Obstacle. Application to Interaction between Dense Snow Avalanches and Defence Structures', Natural Hazards and Earth System Sciences 2, 187-191.

Gray, J. M. N. T., Tai, Y.-C., and Noelle, S.: 2003, 'Shock Waves, Dead-Zones and Particle-Free Regions in Rapid Granular Free Surface Flows', Journal of Fluid Mechanics 491, 161-181.

Hákonardóttir, K. M., Hogg, A. J., Jóhannesson, T., and Tómasson, G.: 2003, 'A Laboratory Study of the Retarding Effect of Braking Mounds on Snow Avalanches', Journal of Glaciology, in press. 
Hákonardóttir, K. M., Jóhannesson, T., Tiefenbacher, F., and Kern, M.: 2001, 'A Laboratory Study of the Retarding Effect of Braking Mounds in 3, 6 and 9 m Long Chutes', Technical report 01007, Icelandic Meteorological Office, Reykjavik, Iceland.

McClung, D. and Schaerer, P.: 1993, The Avalanche Handbook, The Mountaineers, Seattle.

McElwaine, J. and Tiefenbacher, F.: 2003, 'Calculating Internal Avalanche Velocities from Correlation with Error Analysis', Surveys in Geophysics 24, 499-524.

Sparks, R. S. J. et al.: 2002, 'Generation of a Debris Avalanche and Violent Pyroclastic Density Current on 26 December (Boxing Day) 1997 at Soufrière Hills Volcano, Montserrat', in T. H. Druitt and B. P. Kokelaar (eds.), The Eruption of Soufrière Hills Volcano, Montserrat from 1995 to 1999, Geological Society, London, Memoirs, Vol. 21, 409-434.

Tiefenbacher, F. and Kern, M.: 2003, 'Experimental Devices to Determine Snow Avalanche Basal Friction and Velocity Profiles', Cold Reg. Sci. Tech., in press.

Yih, C.-S.: 1979, Fluid Mechanics, West River Press, pp. 144-152. 